\title{
Effects of the energetic-ion-driven instabilities on plasma heating, transport, and rotation in toroidal systems
}

\author{
Ya.I. Kolesnichenko ${ }^{1}$, Yu.V. Yakovenko ${ }^{1}$, V.V. Lutsenko ${ }^{1}$, \\ R.B. White ${ }^{2}$ and A. Weller ${ }^{3}$ \\ ${ }^{1}$ Institute for Nuclear Research, 03680 Kyiv, Ukraine \\ ${ }^{2}$ Princeton Plasma Physics Laboratory, P.O. Box 451, Princeton, NJ 08543, USA \\ ${ }^{3}$ Max-Planck-Institut für Plasmaphysik, IPP-EURATOM Association, D-85748 \\ Garching bei München, Germany
}

\begin{abstract}
A theory is developed from which it follows that energetic-ion-driven instabilities can, first, channel the energy of the energetic ions outside the region where these ions are located and, second, considerably affect the electron heat flux across the magnetic field. A new mechanism of the frequency chirping is revealed. Namely, it is shown that instabilities caused by the energetic ions can influence the plasma rotation, in which case the development of the instabilities results in variation of the Doppler shift in time. It is concluded that a key factor responsible for the mentioned phenomena is the local imbalance of the wave emission by energetic ions and the wave absorption by electrons along the radius. On the basis of the developed theory, experiments on the stellarator Wendelstein 7-AS and the spherical torus NSTX, where effects of Alfvénic activity on the plasma temperature were observed, are considered.
\end{abstract}




\section{Introduction}

Energetic ions produced by neutral beam injection, radio-frequency heating, and fusion reactions are typically present in all types of the toroidal fusion devices and often lead to plasma instabilities. It is usually believed that these instabilities can affect confinement of the energetic ions but not the bulk plasma; some of them can be used for plasma diagnostics. At the same time, there are experiments indicating that the role of the energetic-ion-driven instabilities is not restricted to the mentioned issues. In particular, experiments on the stellarator Wendelstein 7-AS (W7-AS) have shown that Alfvén instabilities can considerably reduce the plasma energy and lead to strong thermal crashes (the temperature dropped by up to $30 \%$ during instability bursts in the discharge \#34723) [1], although typically the discharges in W7-AS were very quiescent in their stationary phase. More recent experiments on the spherical torus NSTX have shown that broadening of the electron temperature correlates with Alfvénic activity [2, 3]. In experiments on both W7-AS and NSTX the instabilities affected the heat transport but had no visible influence on the particle transport. In addition, NSTX experiments have shown that the effect of the Alfvénic activity on the plasma heat transport can differ at different locations: the calculated heat conductivity coefficient increases with injected power in the core region and decreases at the periphery $[2,3]$. Another peculiarity of the NSTX data is that the anomalous heat transport correlates only with high frequency instabilities $(0.5 \div 1.1 \mathrm{MHz})$. These facts have raised a fundamental question on the physical mechanisms involved. The first step in the explanation of these phenomena was made in reference [4]. It was found that the finite electric conductivity leads to waveinduced enlargement of the particle deflection from the oscillating flux surfaces, whereas the Coulomb collisions provide random walk of the particles with enlarged steps, which results in anomalous heat conductivity. It was concluded that the effect is largest in the collisional regime, $\nu_{\mathrm{e}}>k_{\|} v_{\mathrm{e}}$, with $\nu_{\mathrm{e}}$ the electron collision frequency, $v_{\mathrm{e}}$ the electron thermal velocity, and $k_{\|}$the longitudinal wave number. It was this regime that took place in the mentioned W7-AS discharge (and in similar discharges), where the plasma density was high $\left(n_{\mathrm{e}} \sim 10^{20} \mathrm{~m}^{-3}\right)$ but the temperature was low $(T<300 \mathrm{eV})$, which enabled the authors to explain the observed thermal crashes. However, the considered mechanism provides weak enhancement of heat transport in plasmas with high temperature and high frequencies instabilities. Therefore, it cannot explain the NSTX observations.

In connection with the mentioned experiments a number of general questions of practical importance arise. Is it typical or very seldom that energetic-iondriven instabilities strongly affect the electron transport properties? What conditions determine the influence of these instabilities on the electron heat flux? Are there other effects of the instabilities on the bulk plasma? To answer these questions, further development of theory, explanation of available experimental data, and new experiments are required.

In a very recent Letter [5] a new phenomenon - channelling of the energy

and momentum during energetic-ion-driven instabilities — was discovered. It was 
demonstrated by means of a simple model that this phenomenon seems can be responsible for the mentioned NSTX observations. This paper represents an extended version of the work [5]; it gives not only more details, but contains a theory of waveinduced convection and anomalous electron heat conductivity, and comparative analysis of experiments on NSTX and Wendelstein 7-AS.

Theory in this work is aimed at the explanation of experimental observations, such as (i) different effects of energetic-ion-driven instabilities on the heat transport and bulk plasma particle transport; (ii) decrease of the electron temperature in the plasma core when the injected power increases in NSTX; (iii) increase of the electron temperature at the periphery during Alfvén instabilities in NSTX; (iv) frequency chirping of an instability in Wendelstein 7-AS.

The paper is organized as follows. A qualitative analysis of the electron transport across the magnetic field and the plasma rotation in the presence of energetic-ion-driven instabilities is carried out in section 2. Based on the quasilinear theory equations averaged over bounce/transit time, equations for the balance of the number of electrons and the electron temperature are derived and analysed in section 3. Experimental observations on W7-AS and NSTX are considered in section 4. The obtained results are summarized in section 5. The width of the wave-particle resonance for Alfvén waves is calculated in Appendix A.

\section{Anomalous transport and plasma rotation caused by energetic-ion-driven instabilities (qualitative consideration)}

We begin with a qualitative analysis of the influence of instabilities caused by energetic ions on the electron transport and plasma rotation.

By analogy with quantum mechanics, we present the energy density of the wave with the frequency $\omega_{\boldsymbol{k}}$ and the wave number $\boldsymbol{k}$ as $W_{\boldsymbol{k}}=\omega_{\boldsymbol{k}} n_{\boldsymbol{k}}$, where $n_{\boldsymbol{k}}$ is the quasiparticle density (which has the dimension of $h / V$, where $h$ is the Planck constant and $V$ is the volume). Then the wave momentum is $\boldsymbol{k} n_{\boldsymbol{k}}$. The quasi-particles are produced with the rate $2 \gamma_{\alpha}$ by the energetic ions and absorbed with the rate $2 \gamma_{\mathrm{e}}$ by the electrons, where $\gamma_{\alpha}=\left\{\widetilde{\boldsymbol{j}}_{\alpha} \cdot \widetilde{\boldsymbol{E}}\right\} / 2 W>0$ and $\gamma_{\mathrm{e}}=\left\{\widetilde{\boldsymbol{j}}_{\mathrm{e}} \cdot \widetilde{\boldsymbol{E}}\right\} / 2 W<0$ represent local contributions to the instability growth rate of the energetic ions and the electrons, respectively, $\boldsymbol{E}$ is the electric field, $\boldsymbol{j}$ is the current density, tilde labels perturbed quantities; $\{\ldots\}$ means time averaging; the instability growth rate is $\gamma^{\text {ins }}=\int \mathrm{d}^{3} x\left(\gamma_{\alpha}+\gamma_{\mathrm{e}}\right) W / \int \mathrm{d}^{3} x W$. Therefore, the force $\boldsymbol{f}_{\sigma}=-2 \gamma_{\sigma \boldsymbol{k}} n_{\boldsymbol{k}} \boldsymbol{k}(\sigma=\alpha, \mathrm{e})$ acts on the particles, which leads to the radial drift of both electrons and energetic particles with the velocity $\propto\left[\boldsymbol{f}_{\sigma} \times \boldsymbol{B}\right]_{r} / e_{\sigma}(\boldsymbol{B}$ is the magnetic field, $e_{\sigma}$ is the electric charge, $r$ is the radial coordinate) [6]. It is clear that in the steady state the total momentum of the energetic ions and the electrons is conserved provided that the wave damping on the bulk plasma ions is negligible. If this balance were valid also locally, the diffusion of the electrons and the energetic ions would be ambipolar automatically [6]. However, in reality the local balance may be violated. In particular, when the destabilized ideal MHD Alfvén mode generates kinetic waves, the 
wave energy can be absorbed by electrons in the region away from the region where the ideal MHD mode is located (which took place in the experiments on W7-AS [4]). The finite radial mode width and the finite orbit width of the energetic ions also contribute to the violation of the local momentum balance. For instance, because of the continuum damping the mode energy and momentum can be dissipated far away from the region of the resonance interaction between the energetic ions and the mode.

Because the number of the energetic particles is small, their radial flux is small and, thus, in any case, the electron particle flux is small, too. However, the heat flux is not restricted by the ambipolarity condition.

To be more specific, let us write the particle fluxes across the magnetic field [6],

$$
\Gamma_{\sigma}^{\mathrm{res}}=\frac{c f_{\sigma \vartheta}}{e_{\sigma} B}=-\frac{2 c}{e_{\sigma} B} \sum_{\boldsymbol{k}} k_{\vartheta} \gamma_{\sigma \boldsymbol{k}} n_{\boldsymbol{k}},
$$

and the following equation for the wave energy density:

$$
\frac{\partial W_{\boldsymbol{k}}}{\partial t}+\nabla \cdot \boldsymbol{S}_{\boldsymbol{k}}=2 \gamma_{\boldsymbol{k}} W_{\boldsymbol{k}}
$$

where $\gamma_{\boldsymbol{k}}=\gamma_{\alpha, \boldsymbol{k}}+\gamma_{\mathrm{e}, \boldsymbol{k}}, \boldsymbol{S}_{\boldsymbol{k}}$ is the energy density flux, $\vartheta$ is the poloidal coordinate, superscript "res" labels fluxes caused by the resonance wave-particle interaction. Using these equations, we obtain, cf. [6]:

$$
\sum_{\sigma} e_{\sigma} \Gamma_{\sigma}^{\mathrm{res}}=-\frac{2 c}{B} \sum_{\boldsymbol{k}} \frac{k_{\vartheta}}{\omega_{\boldsymbol{k}}}\left(\frac{\partial W_{\boldsymbol{k}}}{\partial t}+\nabla \cdot \boldsymbol{S}_{\boldsymbol{k}}\right) .
$$

It follows from equation (3) that in steady state $\sum_{\sigma} e_{\sigma} \Gamma_{\sigma}^{\mathrm{res}}=0$ provided that $\nabla \cdot \boldsymbol{S}=0$, which is the case only when the local balance between the wave emission and absorption takes place. Otherwise, the resonance fluxes are not ambipolar, and the non-resonance fluxes responsible for the RHS of equation (3) provide ambipolarity.

Both the particle flux and the heat flux are determined, in general, by the diffusion / conductivity and the convection caused by the waves. However, hydrodynamic equations which do not take into account the wave-induced convection are often used to model experimental observations. In this case, an effective heat conductivity coefficient, $\chi_{\mathrm{e}}^{\mathrm{ef}}$, can be introduced:

$$
q_{\mathrm{e}}=\chi_{\mathrm{e}}^{\mathrm{ef}} \frac{n_{\mathrm{e}} T}{L},
$$

where $q_{\mathrm{e}}$ is the electron heat flux, $n_{\mathrm{e}}$ and $T$ are the electron density and plasma temperature, respectively, $L$ is a characteristic length of plasma inhomogeneity; the magnitudes of $q_{\mathrm{e}}, n_{\mathrm{e}}$, and $T$ are to be taken from experimental data and numerical calculations. It is clear that when non-diffusive effects dominate the transport, a code ignoring them calculates $\chi_{\mathrm{e}}^{\text {ef }}$ well exceeding the real heat conductivity coefficient. On the other hand, using equations (1) and (4) and assuming $q_{\mathrm{e}}=T \Gamma_{\mathrm{e}}^{\mathrm{res}}$, we can obtain the following estimate for $\chi_{\mathrm{e}}^{\mathrm{ef}}$ :

$$
\frac{\chi_{\mathrm{e}}^{\mathrm{ef}}}{D_{\mathrm{B}}}=32 m \frac{L}{r} \frac{\left|\gamma_{\mathrm{e}}\right|}{\omega} \frac{W}{n_{\mathrm{e}} T},
$$


where $D_{\mathrm{B}}=c T /(16 e B)$ is the Bohm diffusion coefficient, $B$ is the magnetic field, $m$ is the poloidal mode number $\left(k_{\vartheta}=m / r\right)$. For instance, assuming that $\gamma_{\mathrm{e}}$ is as large as $\gamma_{\alpha}$ and taking $\gamma_{\alpha} / \omega=10^{-1}, W / n_{\mathrm{e}} T=10^{-3}, m=20, a / r=5$ with $a$ the plasma radius, $L / a=3$ (flat radial profiles of plasma parameters in the instability region), we obtain $\chi_{\mathrm{e}}^{\mathrm{ef}} \approx D_{\mathrm{B}}$. This estimate just demonstrates that the effect of energetic-iondriven instabilities on the local electron transport can be significant. Note that, strictly speaking, the analysis above is relevant to fluxes of circulating particles; the trapped particle fluxes contain $n q$ ( $n$ is the toroidal mode number, $q$ is the safety factor) instead of $m$ (see section 3). In the case of Alfvén waves, this difference, however, is considerable only when the their frequency is sufficiently high .

One can expect that the development of energetic-ion-driven instabilities will affect the plasma rotation and the equilibrium radial electric field: As we already mentioned, typically the regions where the waves receive the momentum from energetic ions and give this momentum to electrons do not coincide. This electric field will affect the resonance condition and, thus, the resonance fluxes.

Let us consider the process of generation of the rotation in more details, restricting ourselves to a simple model which neglects effects of toroidicity. We proceed from equations of motion for all the plasma components (electrons, ions, and energetic ions). We sum these equations, assuming that the waves interact resonantly only with energetic ions and electrons and taking into account that the number of the energetic ions is small. As a result, we obtain the following equation for the frequency of the poloidal ion rotation, $\Omega_{\mathrm{i} \vartheta}$ :

$$
M_{\mathrm{i}} n_{\mathrm{i}} r \dot{\Omega}_{\mathrm{i} \vartheta}=\sum_{\sigma=\mathrm{e}, \alpha} f_{\sigma \vartheta}+\frac{1}{r^{2}} \frac{\partial}{\partial r} r^{3} \eta_{*} \frac{\partial \Omega_{\mathrm{i} \vartheta}}{\partial r}-\frac{B}{c} j_{r},
$$

where $\eta_{*}=M_{\mathrm{i}} n_{\mathrm{i}} \eta, \eta$ is the plasma viscosity, $\eta \geq \eta^{\mathrm{col}}, \eta^{\text {col }}=0.3 \rho_{\mathrm{i}}^{2} / \tau_{\mathrm{i}}, \rho_{\mathrm{i}}$ the ion Larmor radius, $\tau_{\mathrm{i}}$ the ion collision time, $j_{r}$ is the radial current density, $j_{r}=j_{\mathrm{i} r}+j_{\mathrm{er}}+j_{\alpha r}$, dot over letters denotes the time derivative, $M$ is the particle mass.

When $k_{\vartheta} \gg k_{\varphi}$ ( $\varphi$ is the toroidal coordinate), $f_{\sigma \vartheta} \gg f_{\sigma \varphi}$. Then it is reasonable to assume that $\dot{u}_{\mathrm{i} \vartheta} \gg \dot{u}_{\mathrm{i} \varphi} B_{\vartheta} / B_{\varphi}\left(\boldsymbol{u}_{\mathrm{i}}\right.$ is the hydrodynamic ion velocity), in which case $\dot{u}_{\mathrm{i} \vartheta}=-c \dot{E}_{r} / B$ (but $u_{\mathrm{i} \vartheta} \equiv r \Omega_{\mathrm{i} \vartheta} \neq-c E_{r} / B !$ ). Using this equation for $\dot{u}_{\mathrm{i} \vartheta}$ and the equation $\dot{E}_{r}=-4 \pi j_{r}$, we can write equation (6) in the form:

$$
\left(1+\frac{c^{2}}{v_{\mathrm{A}}^{2}}\right) j_{r}=\sum_{\sigma=\mathrm{e}, \alpha} j_{\sigma r}+\frac{c}{B} \frac{1}{r^{2}} \frac{\partial}{\partial r} r^{3} \eta_{*} \frac{\partial \Omega_{\mathrm{i} \vartheta}}{\partial r},
$$

where $j_{\sigma r}=c f_{\sigma \vartheta} / B, v_{\mathrm{A}}$ is the Alfvén velocity. It follows from equation (7) that the radial current $\left(j_{r}\right)$ is much less, by a factor of $c^{2} / v_{\mathrm{A}}^{2}$, than the partial currents $j_{\alpha r}$ and $j_{\text {er }}$ due to the generation of the ion current

$$
j_{\mathrm{i} r}=-\frac{c}{B} M_{\mathrm{i}} n_{\mathrm{i}} \dot{u}_{\mathrm{i} \vartheta}+\frac{c}{B} \frac{1}{r^{2}} \frac{\partial}{\partial r} r^{3} \eta_{*} \frac{\partial \Omega_{\mathrm{i} \vartheta}}{\partial r},
$$

which almost compensates the current $j_{\alpha r}+j_{\mathrm{er}}$. This implies that the last term (proportional to $j_{r}$ ) in equation (6) is negligible. This conclusion agrees with equation (3) which shows that the sum of the resonance current and adiabatic current vanishes. 
Similarly, one can show that the frequency of the toroidal rotation $\left(\Omega_{\varphi}\right)$ is described by the equation:

$$
M_{\mathrm{i}} n_{\mathrm{i}} R \dot{\Omega}_{\mathrm{i} \varphi}=\sum_{\sigma=\mathrm{e}, \alpha} f_{\sigma \varphi}+\frac{R}{r} \frac{\partial}{\partial r} r \eta_{*} \frac{\partial \Omega_{\mathrm{i} \varphi}}{\partial r},
$$

where $R$ is the major radius of the torus.

Due to the presence of viscosity, there exist steady-state solutions of equations (6) and (9). The characteristic viscosity time is $\tau^{\mathrm{vis}}=(\Delta r)^{2} / \eta$, where $\Delta r$ is the characteristic scale of the process. Taking this into account, we can evaluate the steadystate local rotation velocities as

$$
\frac{u_{\mathrm{i} \vartheta}}{v_{\mathrm{i}}}=\frac{f_{\alpha \vartheta} \tau^{\mathrm{vis}}}{M_{\mathrm{i}} n_{\mathrm{i}} v_{\mathrm{i}}}=2 m \frac{\gamma_{\alpha}}{\omega} \frac{W}{n_{\mathrm{i}} T_{\mathrm{i}}} \frac{v_{\mathrm{i}} \tau^{\mathrm{vis}}}{r}, \quad u_{\mathrm{i} \varphi} \sim u_{\mathrm{i} \vartheta} k_{\varphi} / k_{\vartheta},
$$

where $v_{\mathrm{i}}=\sqrt{T_{\mathrm{i}} / M_{\mathrm{i}}}, u_{\mathrm{i} \varphi}=R \Omega_{\mathrm{i} \varphi}$. One can easily see from here that the rotation velocity can be significant. For instance, taking $m=2, \gamma_{\alpha} / \omega=10^{-2}, W /\left(n_{\mathrm{i}} T_{\mathrm{i}}\right)=10^{-4}$, $\tau^{\mathrm{vis}}=0.1 \mathrm{~s}, v_{\mathrm{i}}=2 \times 10^{7} \mathrm{~cm} \mathrm{~s}^{-1}$ (for $T \approx 1 \mathrm{keV}$ in deuterium plasma), and $r=20 \mathrm{~cm}$, we obtain $u_{\vartheta}=v_{\mathrm{i}}$.

Of course, this rotation superimposes on the rotation caused by other mechanisms.

Let us assume that the energetic ions emit the waves at the radius $r_{\alpha}$, whereas the electrons absorb the waves at $r_{\mathrm{e}}$, with $r_{\alpha}<r_{\mathrm{e}}$. Then we take $f_{\alpha \vartheta}=f_{*} \delta\left(r-r_{\alpha}\right) / r^{2}, f_{\mathrm{e} \vartheta}=$ $-f_{*} \delta\left(r-r_{\mathrm{e}}\right) / r^{2}$, which conserves the global angular momentum: $\int_{0}^{a} \mathrm{~d} r r^{2}\left(f_{\alpha \vartheta}+f_{\mathrm{e} \vartheta}\right)=0$. Setting $\dot{\Omega}_{\mathrm{i} \varphi}=0$ and $j_{r}=0$ in equation (6) and integrating it twice with the boundary conditions $\Omega_{\mathrm{i} \vartheta}(a)=0, \partial \Omega_{\mathrm{i} \vartheta} /\left.\partial r\right|_{r=0}=0$, we obtain the following steady-state solution:

$$
\Omega_{\mathrm{i} \vartheta}= \begin{cases}f_{*} \int_{r_{\alpha}}^{r_{\mathrm{e}}} \frac{\mathrm{d} r}{r^{3} \eta_{*}} & \text { for } r \leq r_{\alpha}, \\ f_{*} \int_{r}^{r_{\mathrm{e}}} \frac{\mathrm{d} r}{r^{3} \eta_{*}} & \text { for } r_{\alpha} \leq r \leq r_{\mathrm{e}} \\ 0 & \text { for } r_{\mathrm{e}} \leq r \leq a .\end{cases}
$$

We observe that there is sheared rotation in the region $r_{\alpha}<r<r_{\mathrm{e}}$ and rigid rotation in the region $0<r<r_{\alpha}$. In a similar way, one can show that the direction of rotation is reversed when $r_{\alpha}>r_{\mathrm{e}}$, see figure 1 .

The considered rotation has an important consequence: it leads to frequency chirping due to the growth of the Doppler shift, $\Delta \omega(t)=\boldsymbol{k} \cdot \boldsymbol{u}(t)=m \Omega_{\vartheta}-n \Omega_{\varphi}$ [we assume $\widetilde{\boldsymbol{E}} \propto \exp (-\mathrm{i} \omega+\mathrm{i} m \vartheta-\mathrm{i} n \varphi)$ ], after the beginning of the instability. One can see that the effect is strongest when the region of the wave emission by the energetic ions lies at smaller radii than the region of the wave absorption, see figure 1 . In this case, $\operatorname{sgn} u_{\vartheta}=\operatorname{sgn} f_{\alpha \vartheta}=-\operatorname{sgn} k_{\vartheta}$ and $\operatorname{sgn} u_{\varphi}=\operatorname{sgn} f_{\alpha \varphi}=-\operatorname{sgn} k_{\varphi}$; therefore, $\Delta \omega<0$, i.e., frequency chirping down takes place.

Note that although we have used a very simple model to describe the plasma rotation caused by instabilities, one can expect that our qualitative conclusions will be true in real situations. In particular, even if the velocity of the poloidal rotation were considerably reduced, which may be the case in tokamaks, the Doppler shift due to $u_{\vartheta} \neq 0$ can be considerable for the modes located close to the magnetic axis. On the 

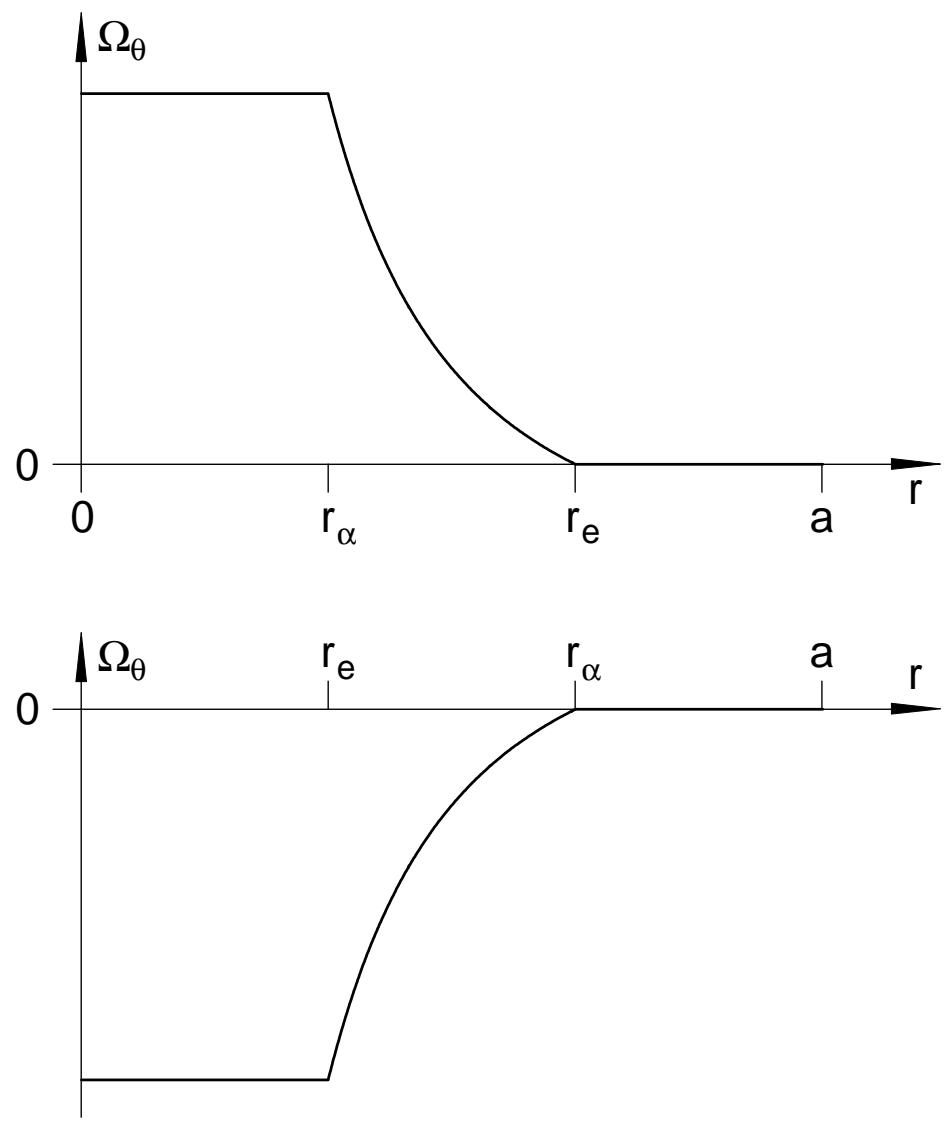

Figure 1. Sketch of the plasma rotation during energetic-ion-driven instabilities: upper graph, $r_{\alpha}<r_{\mathrm{e}}$; lower graph, $r_{\alpha}>r_{\mathrm{e}}$.

other hand, the Doppler shift due to toroidal rotation can also be important, especially in toroidal plasmas with small aspect ratio.

\section{Energy channelling and electron heat transport (quasilinear theory)}

In this section we study the electron transport caused by the energetic-ion-driven instabilities in more details. In addition, we consider the energy channelling - a new phenomenon which can strongly affect the electron temperature and, thus, the plasma energy balance without direct influence on the electron transport.

Using quasilinear theory equations averaged over the particle bounce / transit time $\left(\tau_{\mathrm{b}}\right)$ (see, e.g., reference [7]), we can write:

$$
\frac{\partial F}{\partial t}=\frac{1}{\tau_{\mathrm{b}}} \sum_{m, n}\left(\frac{\partial}{\partial \mathcal{E}}-\frac{n}{\omega} \frac{\partial}{\partial P_{\varphi}}\right) \tau_{\mathrm{b}} \mathcal{D}\left(\frac{\partial}{\partial \mathcal{E}}-\frac{n}{\omega} \frac{\partial}{\partial P_{\varphi}}\right) F+C^{\mathrm{COL}},
$$

where $F=F\left(\mathcal{E}, \mu, P_{\varphi}, t\right)$ is the electron unperturbed distribution function, $\mathcal{E}$ is the particle energy, $\mu$ is the magnetic moment, $P_{\varphi}=M v_{\|} R-e \psi_{\mathrm{p}} / c$ is the canonical angular momentum, $\psi_{\mathrm{p}}$ is the poloidal magnetic flux, $v_{\|}$is the particle velocity along 
the magnetic field, $C^{\mathrm{COL}}$ is the collision term,

$$
\mathcal{D}=\pi e^{2} \sum_{s}|\mathcal{J}|^{2} \delta\left(\omega-m\langle\dot{\vartheta}\rangle+n\langle\dot{\varphi}\rangle-s \omega_{\mathrm{b}}\right),
$$

$\mathcal{J}$ is proportional to wave amplitudes and depends on particle orbits in the equilibrium magnetic field, $\omega_{\mathrm{b}}=2 \pi / \tau_{\mathrm{b}}$, brackets denote bounce averaging, $n$ is the toroidal mode number, $\delta$ is the Dirac delta function, the subscript "e" is omitted for brevity. The perturbations are taken in the form $\tilde{X}=\hat{X}\left(P_{\varphi}\right) \exp (-\mathrm{i} \omega t-\mathrm{i} n \varphi+\mathrm{i} m \vartheta)$, and a righthanded coordinate system is used. The equations (12) and (13) are written in the assumption that the wave frequency is small in comparison to the electron gyrofrequency. The reason for this assumption is that the frequencies of the waves destabilized by the energetic ions are normally even less than the ion gyrofrequency.

To describe the radial fluxes of the particles and the energy, we neglect the electron orbit width in $P_{\varphi}$ and proceed to the variables $\mathcal{E}, \mu$, and $r$, with $r$ the flux surface radius. Then the equation for the well circulating particles can be obtained from equation (12) by replacing $n \partial / \partial P_{\varphi}$ with $-\left(m / M \omega_{B} r\right) \partial / \partial r$, where $\omega_{B}$ is the gyrofrequency. The equation for the deeply trapped particles will contain $n q$ ( $q$ is the safety factor) instead of $m$ in the same terms. This difference is negligible for the waves with $k_{\|} R \equiv m q^{-1}-n \ll 1$ but important when $k_{\|}$is larger. Keeping this in mind, below we neglect the difference between $m$ and $n q$ in order to write only one quasilinear equation valid for both circulating particles and trapped ones. Note that the same conclusion concerning different mode numbers ( $m$ or $n$ ) in the equations for the circulating particles and the trapped ones can be drawn by considering the motion of individual particles in the wave field.

Equation (12) describes the change of $F$ due to the wave-particle interaction through the resonance included in equation (13), which implies that it ignores effects which are not associated with this resonance. Taking this into account, we present the particle radial flux, $\Gamma_{\mathrm{e}}$, as follows:

$$
\Gamma_{\mathrm{e}}=\Gamma_{\mathrm{e}}^{\mathrm{res}}(1-\mathcal{N})
$$

where $\Gamma_{\mathrm{e}}^{\mathrm{res}}$ is the particle flux which can be obtained by using equation (12), $\mathcal{N}$ is the factor describing a possible reduction of the flux $\Gamma_{\mathrm{e}}^{\mathrm{res}}, \mathcal{N}$ may vary from 0 to 1 . For instance, it is known that high frequency waves associated with electrons only (i.e., waves which do not affect the ion motion) cannot lead to anomalous diffusion but can influence heat conductivity [8]. In this case $\Gamma_{\mathrm{e}}=0$ and, thus, $\mathcal{N}=1$. The generation of the radial electric field which affects the resonance condition can also result in $\mathcal{N}=1[4]$. In contrast to this, $\mathcal{N}=0$ when the energetic particles excite such instabilities that local balance, $\Gamma_{\alpha}^{\mathrm{res}}(r)=\Gamma_{\mathrm{e}}^{\mathrm{res}}(r)$ takes place, see section 2 .

In order to calculate $\Gamma_{\mathrm{e}}^{\mathrm{res}}$, we integrate equation (12) over the velocity space. When doing this, we take into account that the velocity volume element averaged over the flux surface is $\overline{\mathrm{d}^{3} v}=C \sum_{\sigma} \mathrm{d} \mathcal{E} \mathrm{d} \mu \tau_{\mathrm{b}}$, with $C=B /(M q R), \sigma$ the sign of the particle velocity, the summation over $\sigma$ being absent for the trapped particles. We obtain:

$$
\frac{\partial n_{\mathrm{e}}}{\partial t}+\frac{1}{r} \frac{\partial}{\partial r} r \Gamma_{\mathrm{e}}^{\mathrm{res}}(1-\mathcal{N})=0
$$


where $n_{\mathrm{e}}=\int \overline{\mathrm{d}^{3} v} F$, and

$$
\Gamma_{\mathrm{e}}^{\mathrm{res}}=-\sum_{m, n} \frac{k_{\vartheta}}{M \omega_{B} \omega} \int \overline{\mathrm{d}^{3} v} \mathcal{D}\left(\frac{\partial}{\partial \mathcal{E}}+\frac{k_{\vartheta}}{M \omega_{B} \omega} \frac{\partial}{\partial r}\right) F,
$$

$k_{\vartheta}=m / r$. The first term in equation (16) represents the convective flux, whereas the second term represents the diffusive flux. Equation (16) shows that if the wave spectrum produced by the energetic ions were symmetric with respect to $k_{\vartheta}$, the convective term would vanish. However, normally the spectrum is not symmetric; furthermore, only a single mode may be destabilized. When the instability is excited due to spatial inhomogeneity of the energetic ions (which implies that the mode rotates in the ion diamagnetic direction), $m<0, n<0$ and, thus, $\Gamma_{\mathrm{e}}^{\mathrm{res}}$ determined by equation (16) is positive (note that $\omega_{B}<0$ ). When the instability is excited because of the velocity anisotropy or due to inverted energy distribution of the energetic ions, the sign of the mode numbers can be either negative or positive, and the fluxes of the energetic ions and the electrons are directed either outwards or inwards (depending on the direction of the beam ions).

Calculation of the second moment of equation (12) and combination of the obtained equation with equation (15) yields:

$$
\frac{3}{2} n_{\mathrm{e}} \frac{\partial T_{\mathrm{e}}}{\partial t}+\frac{1}{r} \frac{\partial}{\partial r} r q_{\mathrm{e}}=Q_{\mathrm{e}}+Q^{\mathrm{COL}}
$$

where $T_{\mathrm{e}}=(1 / 3) \int \overline{\mathrm{d}^{3} v} M v^{2} F, Q^{\mathrm{COL}}$ is the energy exchange of electrons and other particles through Coulomb collisions, $Q_{\mathrm{e}}$ describes the absorption of the wave energy by electrons,

$$
Q_{\mathrm{e}}=-\sum_{m, n} \int \overline{\mathrm{d}^{3} v} \mathcal{D}\left(\frac{\partial}{\partial \mathcal{E}}+\frac{k_{\vartheta}}{M \omega_{B} \omega} \frac{\partial}{\partial r}\right) F+Q_{\mathrm{e}}^{0}
$$

$q_{\mathrm{e}}$ is the heat flux given by

$q_{\mathrm{e}}=-\sum_{m, n} \frac{k_{\vartheta}}{M \omega_{B} \omega} \int \overline{\mathrm{d}^{3} v}\left[\mathcal{E}-\frac{3}{2}(1-\mathcal{N}) T_{\mathrm{e}}\right] \mathcal{D}\left(\frac{\partial}{\partial \mathcal{E}}+\frac{k_{\vartheta}}{M \omega_{B} \omega} \frac{\partial}{\partial r}\right) F+q_{\mathrm{e}}^{0}$,

where $Q_{\mathrm{e}}^{0}$ and $q_{\mathrm{e}}^{0}$ are contributions to the electron heating and electron heat flux due to Coulomb collisions and effects which are not described by equation (12), in particular, the influence of the radial electric field on the resonance condition.

It follows from equation (19) that the waves affect the electron heat flux even in the case of $\mathcal{N} \approx 1$, i.e., when $\Gamma_{\mathrm{e}} \approx 0$. On the other hand, we observe that equations (16) and (18) contain the same integral. Therefore, when the diffusion is ambipolar due to the generation of the radial electric field under the influence of the destabilized monochromatic wave [4], $\Gamma_{\mathrm{e}}$ and the wave-induced part of $Q_{\mathrm{e}}\left(Q_{\mathrm{e}}^{\mathrm{w}}\right)$ vanish simultaneously. However, $Q_{\mathrm{e}}^{\mathrm{w}} \neq 0$ when several waves are destabilized simultaneously.

One can see that the $Q_{\mathrm{e}}$-term well exceeds the $\nabla \cdot \boldsymbol{q}_{\mathrm{e}}$ term in equation (17) when $\omega \gg \omega_{* \mathrm{e}}$, where $\omega_{* \mathrm{e}}$ is the electron diamagnetic drift frequency. This implies that the main effect of high-frequency waves is plasma heating, whereas wave-induced transport processes play a minor role in establishing the temperature. 


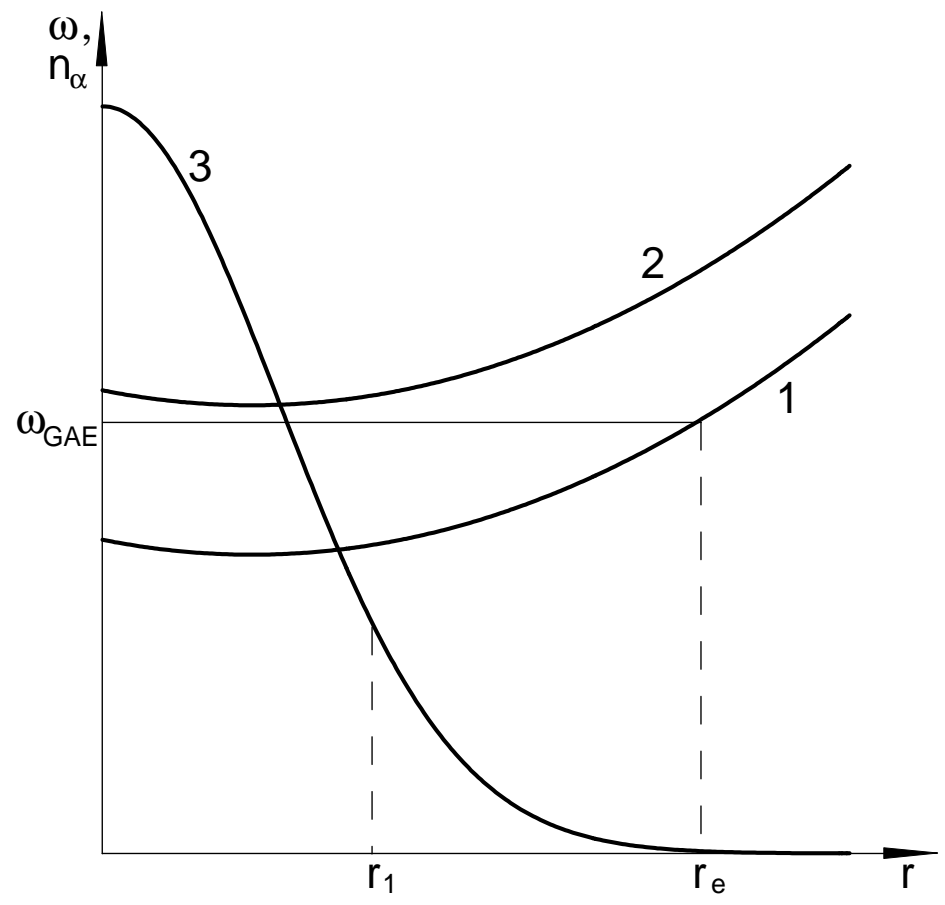

Figure 2. GAE frequency (horizontal line), Alfvén continuum branches with the mode numbers $m, n$ and $m+1, n$ (curves 1,2 ), and the radial profile of the beam ions (curve 3). This sketch demonstrates the energy channelling by a GAE mode: the mode receives the energy of the beam ions mainly inside the region $r<r_{1}$ but gives the energy to electrons due to the continuum damping at $r \approx r_{\mathrm{e}}$.

When the plasma region heated by the waves is located away from the region where the waves receive the energy of the energetic ions, one can say that the destabilized modes channel the energy of the energetic ions from one region to another one. For instance, this can be the case when beam particles with a peaked radial distribution destabilize high-frequency GAE modes, so that the main mechanism of the wave damping is the continuum damping at the periphery, see figure 2. Of course, this effect is significant provided that the power density received by the waves from the beam ions is considerable. If the waves receive a major part of the power injected into the plasma core, the energy channelling will lead to a decrease of the temperature at the plasma centre and an increase at the periphery. In other words, the energy channelling plays an important role when the magnitude of $2 \gamma_{\alpha} W$ is comparable to the injected power density and the energy range of the resonance wave-particle interaction is wide, from an energy close to the birth energy $\left(\mathcal{E}_{0}\right)$ to $\mathcal{E} \ll \mathcal{E}_{0}$.

The slowing down process is accompanied by radial motion of the ion (outwards when $m<0$, i.e., when the modes rotate in the ion diamagnetic direction, and inwards when $m>0$ ). However, the ion displacement is very small at high $\omega$, as follows from the characteristics of the quasilinear equation:

$$
\mathrm{d} r^{2}=\frac{2 m}{M_{\alpha} \omega_{B \alpha} \omega} \mathrm{d} \mathcal{E}
$$

Note that equation (20) does not depend on the particle mass, but depends on the 
electric charge. Therefore, it follows from this equation the acceleration of the electrons due to the wave damping leads to the electron motion outwards when $m<0$. This means that flattening of $F(r)$ during quasilinear evolution tends to increase the electron temperature at the periphery.

Let us consider equation (19) for the heat flux in more details. We observe that the heat flux, like the particle flux, is determined by both convection and diffusivity (the diffusivity term is responsible for the anomalous heat conductivity):

$$
q_{\mathrm{e}}=q_{\mathrm{e}}^{\text {conv }}+q_{\mathrm{e}}^{\text {conduct }}
$$

where $q_{\mathrm{e}}^{\text {conv }}$ is the first term (proportional to $\partial / \partial \mathcal{E}$ ) in equation (19) and $q_{\mathrm{e}}^{\text {conduct }}$ is the second term (proportional to $\partial / \partial r$ ) in this equation. The ratio of $q_{\mathrm{e}}^{\text {conduct }} / q_{\mathrm{e}}^{\text {conv }}$ is small (unless the wave spectrum is symmetric, in which case the convective term vanishes) when $\omega \gg \omega_{* \mathrm{e}}$. The convective flux decreases the electron temperature $\left(\nabla \cdot \boldsymbol{q}_{\mathrm{e}}^{\text {conv }}>0\right)$ in the region where $\partial \mathcal{D} / \partial r>0$ and increases the temperature $\left(\nabla \cdot \boldsymbol{q}_{\mathrm{e}}^{\text {conv }}<0\right)$ in the region with $\partial \mathcal{D} / \partial r<0$. This implies that when the mode is located in the region $r_{\min }<r<r_{\max }$, the convection deteriorates the energy confinement at $r \gtrsim r_{\min }$ and improves the confinement at $r \lesssim r_{\max }$ (although $q_{\mathrm{e}}^{\text {conv }}(r) \geq 0$ everywhere). When $\mathcal{D}$ weakly depends on $r$ inside the region of the mode location, $\nabla \cdot \boldsymbol{q}_{\mathrm{e}}^{\text {conv }}$ is small, and the transport is determined by the conductivity. The convection plays an important role in regions with considerable gradients of the mode energy $\left(r \gtrsim r_{\min }\right.$ and $\left.r \lesssim r_{\max }\right)$. The described effect of the convection is demonstrated in figure 3 .

Now we define the heat conductivity coefficient, $\chi_{\mathrm{e}}$, by means of the equation $q_{\mathrm{e}}^{\text {conduct }}=-n_{\mathrm{e}} \chi_{\mathrm{e}} \partial T / \partial r$. Then, assuming that $\mathcal{N} \approx 1$ and using equations (13), (19) and (21), we can write the following estimate:

$$
\chi_{\mathrm{e}} \approx \sum_{m, n, s} \frac{\pi k_{\vartheta}^{2} c^{2}}{B^{2} \omega^{2} n_{\mathrm{e}} T_{\mathrm{e}}} \int \overline{\mathrm{d}^{3} v} \mathcal{E}|\mathcal{J}|^{2} \delta\left(\omega-m\langle\dot{\vartheta}\rangle+n\langle\dot{\varphi}\rangle-s \omega_{\mathrm{b}}\right) F .
$$

Note that when the unperturbed distribution function is close to Maxwellian, then $q_{\mathrm{e}}^{\text {conv }} \approx\left(\omega / \omega_{* \mathrm{e}}\right) q_{\mathrm{e}}^{\text {conduct }}$. Therefore, knowing $\chi_{\mathrm{e}}$ and, thus, $q_{\mathrm{e}}^{\text {conduct }}$, one can evaluate the convective flux as

$$
q_{\mathrm{e}}^{\mathrm{conv}} \sim \frac{M \omega_{B} \omega n_{\mathrm{e}}}{k_{\vartheta 0}} \chi_{\mathrm{e}}
$$

where $k_{\vartheta 0}$ is a characteristic $k_{\vartheta}$.

We evaluate first $\chi_{\mathrm{e}}$ for the circulating particles. Taking into account that these particles interact with waves mainly through the main resonances $(s=0)$ and the sideband resonances with $s= \pm 1$ (we assume that the poloidal wave length well exceeds the electron orbit width) and using the results of reference [7], we can write:

$$
\mathcal{J}^{\mathrm{c}}=\frac{v_{\mathrm{D}}}{2}\left|\widetilde{E}_{\perp}\right| \delta_{s, \pm 1}+v_{\|} \widetilde{E}_{\|} \delta_{s, 0},
$$

where $\widetilde{\boldsymbol{E}}_{\perp}$ and $\widetilde{E}_{\|}$are components of the wave electric field across and along the equilibrium magnetic field, respectively, $v_{\mathrm{D}}$ is the particle drift velocity, the superscript "c" labels circulating particles, $\delta_{i, j}$ is the Kronecker symbol. The longitudinal electric 


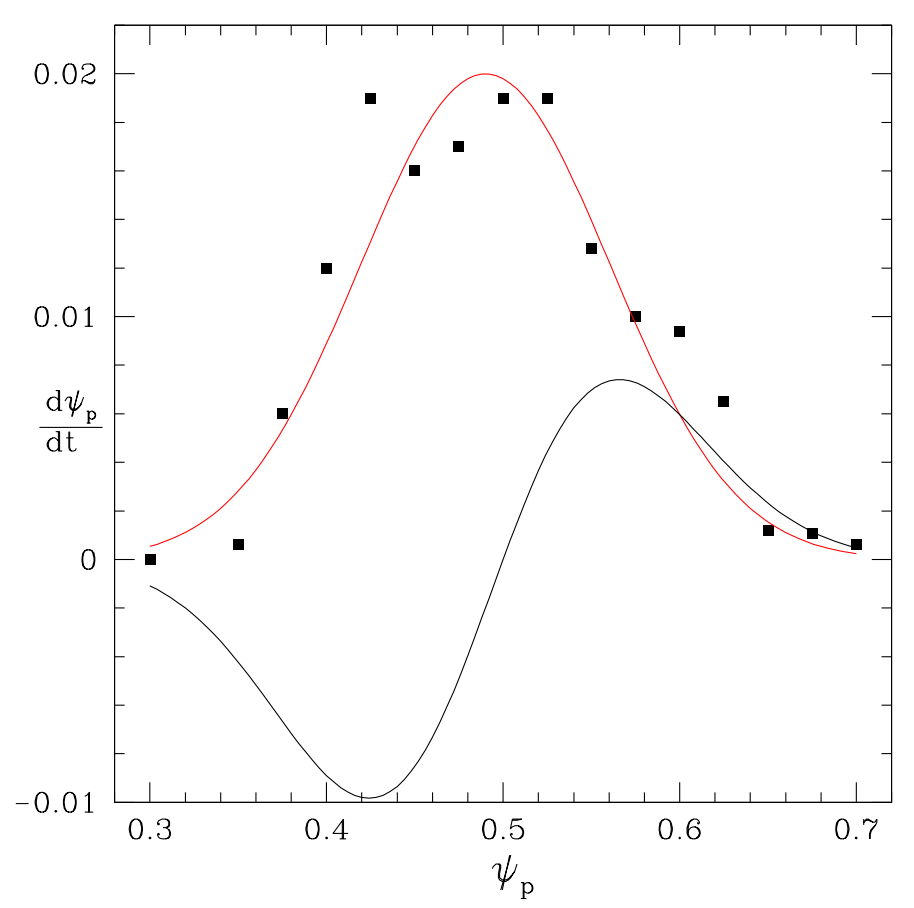

Figure 3. Electron convection caused by a mode located in the middle of the plasma radius. The upper curve represents $q_{\mathrm{e}}(r)$; the lower curve, $-\mathrm{d} q_{\mathrm{e}} / \mathrm{d} r$. We observe that $q_{\mathrm{e}}(r)>0$, but $\mathrm{d} q_{\mathrm{e}} / \mathrm{d} r<0$ in the region where the mode amplitude decreases with radius, which means that the plasma is heated in this region. A standard NSTX equilibrium with the magnetic field on the axis $B=0.45 \mathrm{~T}$ was used, the mode was described by $\widetilde{\boldsymbol{B}}=\nabla \times\left(\alpha_{B} \boldsymbol{B}\right)$, where $\alpha_{B}=\alpha_{m n}\left(\psi_{\mathrm{p}}\right) \sin (n \varphi-m \vartheta), m / n=20 / 20$, $\alpha_{m n}\left(\psi_{\mathrm{p}}\right)$ was a Gaussian, $\psi_{\mathrm{p}}$ the poloidal magnetic flux, $\alpha_{\max }=10^{-4}$, and the mode frequency is $778 \mathrm{kHz}$. There were 20000 of $30-\mathrm{keV}$ electrons, initially all at one surface. Calculation was carried out by the code ORBIT.

field of Alfvén waves is small. However, the electron drift velocity is small, too. Therefore, it is not clear which term in equation (24) is the most important. To compare them, we note that

$$
\widetilde{E}_{\|}=-k_{\perp} k_{\|} \rho_{\mathrm{s}}^{2} \widetilde{E}_{\perp}
$$

where $\rho_{\mathrm{s}}=c_{s} / \omega_{B i}$, with $c_{\mathrm{s}}=\sqrt{T_{\mathrm{e}} / M_{\mathrm{i}}}$. Then we conclude that the $\widetilde{E}_{\|}$term dominates provided that

$$
2 \sqrt{\frac{M_{\mathrm{i}}}{M_{\mathrm{e}}}} k_{\|} k_{\perp} R \rho_{\mathrm{s}} \gg 1
$$

Equation (26) is normally satisfied and, thus, the resonance $\omega=k_{\|}\left\langle v_{\|}\right\rangle$is mainly responsible for the heat transport by circulating electrons. Keeping only the $s=0$ term in equation (22), we obtain:

$$
\chi_{\mathrm{e}}^{\mathrm{c}} \approx \sum_{m, n} \frac{1}{4 \epsilon^{2}} \frac{k_{\vartheta}^{2}}{k_{\|}^{2}}\left(\frac{\omega}{k_{\|} v_{\mathrm{e}}}\right)^{4} \frac{c^{2}}{k_{\|} v_{\mathrm{e}}} \frac{\left|\widetilde{E}_{\|}\right|^{2}}{B_{0}^{2}},
$$

where $v_{\mathrm{e}}=\sqrt{T_{\mathrm{e}} / M_{\mathrm{e}}}, \epsilon=r / R$. 
In contrast to the circulating electrons, the trapped electrons effectively interact with waves through many resonances corresponding to various $s$. Due to this, particles in a wide energy range can contribute to the transport. To calculate the diffusivity coefficient, we replace the sum over $s$ with an integral in equation (13). As in the case of the circulating particles, we take into account only the longitudinal electric field, $\widetilde{E}_{\|}$. One can show that this is justified when

$$
\frac{s}{3} \sqrt{\frac{M_{\mathrm{i}}}{M_{\mathrm{e}}}} k_{\perp} \rho_{\mathrm{s}} \gg 1
$$

which, typically, is satisfied. To obtain this equation, we have taken into account that the $\widetilde{E}_{\|}$term in $\mathcal{J}^{\text {t }}$ equals $s \omega_{\mathrm{b}} / k_{\|}$, whereas the $\widetilde{E}_{\perp}$ term is of the order $v_{\mathrm{D}}[7]$. Then $\mathcal{J}^{\mathrm{t}}=\widetilde{E}_{\|} J_{s}(z) \omega / k_{\|}$, with $J_{s}(z)$ the Bessel function, $z=2 \kappa k_{\|} q R$, and $\kappa$ the particle trapping parameter. The high frequency instability observed in NSTX $[2,3]$ had $k_{\|} q R \gg 1$. Assuming that this condition is satisfied, we approximate the Bessel function as $J_{s}^{2}(z)=(2 / \pi) \cos ^{2}(\ldots) / \sqrt{z^{2}-s^{2}}[12]$. Then we obtain from equation (22) the trapped electron conductivity coefficient as follows:

$\chi_{\mathrm{e}}^{\mathrm{t}}=\sum_{m, n} \frac{n^{2} q^{2} c^{2}\left|\widetilde{E}_{\|}\right|^{2}}{r^{2} k_{\|}^{2} B^{2} n_{\mathrm{e}} T_{\mathrm{e}}} \int \overline{\mathrm{d}^{3} v} \frac{\mathcal{E} F}{\sqrt{\left(2 \kappa k_{\|} q R \omega_{\mathrm{b}}\right)^{2}-\omega^{2}}} \approx \sum_{m, n} \frac{n^{2} q^{2} c^{2}}{r^{2} k_{\|}^{3} v_{\mathrm{e}}} \frac{\left|\widetilde{E}_{\|}\right|^{2}}{B^{2}} \Lambda$,

where $\Lambda=\ln \left(\alpha+\sqrt{\alpha^{2}-1}\right)$, and $\alpha=2 k_{\|} v_{\mathrm{e}} \sqrt{\epsilon} / \omega$.

Quasilinear theory is based on the assumption that resonances corresponding to various wave numbers overlap, in which case collisionless particle motion is stochastic. Nevertheless, one can use this theory even in the case of a monochromatic wave provided that the particle motion is stochastic for some reason (due to overlap of bounce resonances, Coulomb collisions etc.). Let us obtain first a condition of stochasticity for collisionless trapped particles in a monochromatic wave. The resonance velocity $v_{s}$ corresponding to the bounce resonance with the number $s$ is determined by the equation $\omega=s \omega_{\mathrm{b}}\left(v_{s}\right)$. This leads to $\Delta v \equiv v_{s}-v_{s+1}=v_{s} /(s+1)$. On the other hand, the width of the resonance island (resonance width) in the presence of Alfvén waves can be evaluated by taking $\delta v^{\text {res }}=\delta v_{\|}^{\text {res }} / \sqrt{\epsilon}$, where $\delta v_{\|}^{\text {res }}$ is (see reference [4] and Appendix A for details):

$$
\delta v_{\|}^{\text {res }}=4 \sqrt{e E_{\|} /\left(k_{\|} M\right)}
$$

Now we can write:

$$
\mathcal{K}^{t} \equiv \frac{\delta v^{\mathrm{res}}}{\Delta v}=(s+1) \frac{\delta v^{\mathrm{res}}}{v_{s}}=\frac{2 \sqrt{2}(s+1)}{k_{\|} q R} \frac{\omega_{\mathrm{w}}}{\omega_{\mathrm{bs}}^{t}}>1,
$$

where $\omega_{\mathrm{b} s}^{\mathrm{t}}=v_{s} \sqrt{\epsilon} /(\sqrt{2} q R)$ is the bounce frequency of the deeply trapped electrons with the velocity $v_{s}, \omega_{\mathrm{w}}=k_{\|} \delta v_{\|}^{\text {res }}$. Equation (31) is in qualitative agreement with the stochasticity condition obtained in reference [13] with a more rigorous procedure. It is satisfied for sufficiently large $s$. However, it can hardly be satisfied for the $s=1$ resonance which provides interaction of waves with trapped particles having the highest energy. Therefore, it is of interest to obtain a stochasticity condition for the case when several waves are destabilized. Assuming that $\omega=k_{\|} v_{\mathrm{A}}$, let us consider 
waves with the longitudinal wave numbers $k_{\| 1}$ and $k_{\| 2}$. Particles interacting with these waves through a bounce resonance with given $s$ have such velocities $v_{1}^{\text {res }}$ and $v_{2}^{\text {res }}$ that $v_{2}^{\text {res }}-v_{1}^{\text {res }}=v_{1}^{\text {res }}(\Delta m-q \Delta n) /\left(k_{\| 1} q R\right)$, where $\Delta m=m_{2}-m_{1}$ and $\Delta n=n_{2}-n_{1}$. Then for a particular case of $\Delta m=1, \Delta n=0$ we obtain:

$$
\mathcal{K}^{\mathrm{t}}=k_{\| 1} q R \frac{\delta v^{\mathrm{res}}}{v_{1}^{\mathrm{res}}}>1
$$

This condition can be satisfied when $k_{\|} q R \gg 1$.

Collisionless motion of the circulating particles interacting with the waves mainly through the $s=0$ resonance can be stochastic only in the presence of waves with various mode numbers. In this case, the finite radial mode width is of importance. Let us assume that modes with the numbers $m$ and $m+1$ are located around the radii $r_{1}$ and $r_{2}$, respectively, and that $r_{12} \equiv\left|r_{2}-r_{1}\right|$ is less than the mode width, so that these modes overlap. Using the resonance condition $\omega=k_{\|} v_{\|}$and taking $\omega=k_{\|} v_{\mathrm{A}}$, we can write the resonance velocities at $r=r_{2}$ as follows:

$$
\begin{aligned}
& v_{2}^{(1)}=\frac{v_{\mathrm{A} 1}}{1+\frac{k_{1}^{(1)^{\prime}}}{k_{1}^{(1)}} r_{12}+\frac{k_{1}^{(1)^{\prime \prime}}}{2 k_{1}^{(1)}} r_{12}^{2}}, \\
& v_{2}^{(2)}=v_{\mathrm{A} 2}=v_{\mathrm{A} 1}\left(1+\frac{v_{\mathrm{A} 1}^{\prime}}{v_{\mathrm{A} 1}} r_{12}+\frac{v_{\mathrm{A} 1}^{\prime \prime}}{2 v_{\mathrm{A} 1}} r_{12}^{2}\right),
\end{aligned}
$$

where superscripts "(1)" and "(2)" label the resonant parallel velocity in the field of the modes with the numbers $m$ and $m+1$, respectively, the subscripts " 1 " and "2" refer to the points $r_{1}$ and $r_{2}, k_{1,2} \equiv k_{\|}\left(r_{1,2}\right)$, prime denotes the radial derivative. Then

$$
\Delta v_{\|}^{\mathrm{c}} \equiv v_{2}^{(2)}-v_{2}^{(1)}=v_{\mathrm{A} 1}\left[\frac{v_{\mathrm{A} 1}^{\prime \prime}}{2 v_{\mathrm{A} 1}}-\left(\frac{v_{\mathrm{A} 1}^{\prime}}{v_{\mathrm{A} 1}}\right)^{2}+\frac{k_{1}^{\prime \prime}}{2 k_{1}}\right] r_{12}^{2} \equiv \frac{r_{12}^{2}}{L_{\mathrm{ef}}^{2}} v_{\mathrm{A} 1},
$$

where $L_{\mathrm{ef}}$ is a characteristic length of inhomogeneity. When $\Delta v_{\|}^{\mathrm{c}}<\delta v_{\|}^{\mathrm{res}}$, the particles with $v_{\|} \approx v_{\mathrm{A}}$ located in the interval $r_{1} \lesssim r \lesssim r_{2}$ are in resonance with both waves. This condition is easily satisfied when $r_{12}$ is sufficiently small.

Stochasticity conditions for marginally circulating and marginally trapped particles are milder.

Note that although the obtained equations for the heat flux are general, particular estimates of the heat conductivity coefficients [equations (27) and (29)] are made in the assumption that the velocity distribution is Maxwellian. This assumption is justified provided that the collision term dominates in equation (12). Let us see when this can be the case. We evaluate the quasilinear term [the first term in equation (12)] and the collisional term for the trapped particles as follows:

$$
\begin{aligned}
C^{\mathrm{QL}} & \sim \mathcal{D} \frac{F}{(\Delta \mathcal{E})_{\mathrm{QL}}^{2}} \sim \frac{e^{2} \omega^{2} \widetilde{E}_{\|}^{2} F}{k_{\|}^{3} v_{\mathrm{e}}(\Delta \mathcal{E})_{\mathrm{QL}}^{2}}, \\
C^{\mathrm{COL}} & \sim \nu_{\mathrm{e}} T_{\mathrm{e}}^{2} \frac{F}{(\Delta \mathcal{E})_{\mathrm{QL}}^{2}},
\end{aligned}
$$


where $(\Delta \mathcal{E})_{\mathrm{QL}}$ and $(\Delta v)_{\mathrm{QL}}$ represent the width of the resonance region. It follows from equations (36), (37) that the condition $C^{\mathrm{COL}} \gg C^{\mathrm{QL}}$ for the trapped particles can be written as

$$
\frac{\nu_{\mathrm{e}}}{k_{\|} v_{\mathrm{e}}} \gg \epsilon^{2}\left(\frac{\delta v^{\mathrm{res}}}{4 v_{\mathrm{e}}}\right)^{4} \frac{v_{\mathrm{A}}^{2}}{v_{\mathrm{e}}^{2}} .
$$

In the case of the circulating particles we have:

$$
\begin{aligned}
C^{\mathrm{QL}} & \sim \frac{\pi e^{2} E_{\|}^{2} F}{M^{2} v_{r}\left(\Delta v_{\|}\right)_{\mathrm{QL}}^{2} \Delta k_{\|}}, \\
C^{\mathrm{COL}} & \sim \nu_{\mathrm{e}} \frac{v_{\mathrm{e}}^{2}}{\left(\Delta v_{\|}\right)_{\mathrm{QL}}^{2}} F,
\end{aligned}
$$

where $\left(\Delta v_{\|}\right)_{\mathrm{QL}}$ is the width of the resonance region. We obtain from here that the distribution function of the circulating electrons is close to Maxwellian provided that

$$
\frac{\nu_{\mathrm{e}}}{k_{\|} v_{\mathrm{e}}} \gg\left(\frac{\delta v_{\|}^{\mathrm{res}}}{4 v_{\mathrm{e}}}\right)^{3} \frac{\delta v_{\|}^{\mathrm{res}}}{\left(\Delta v_{\|}\right)_{\mathrm{QL}}} .
$$

In a particular case of monochromatic wave $\left(\Delta v_{\|}\right)_{\mathrm{QL}}=\delta v_{\|}^{\text {res }}$. Then equation (41) is reduced to the condition of plateau regime of reference [4], and the heat conductivity coefficient given by equation (27) for $v_{\mathrm{e}} \sqrt{\epsilon}=v_{\|}=\omega / k_{\|}$coincides with the corresponding coefficient obtained in reference [4] for the plateau regime.

\section{Analysis of experimental observations on W7-AS and NSTX}

In this section we apply the developed theory to experiments on W7-AS and NSTX, where Alfvénic activity affected the electron heat transport.

We begin our analysis with the comparison of experimental observations in these devices. First of all, we note that only high frequency instabilities in the range of $f \equiv \omega / 2 \pi \sim 0.5 \div 1.1 \mathrm{MHz}$, which were identified as GAE modes [10], influenced the electron heat transport in NSTX [2]; the observed instabilities in the range of $0 \div 200 \mathrm{kHz}$ had no visible influence on the electrons. The multiple GAEs resulted in flattening of the electron temperature in NSTX, the effect growing with the injected power $(2 \mathrm{MW}$, $4 \mathrm{MW}$, and $6 \mathrm{MW}$ in the discharges \#120442, \#120434, and \#120438, respectively) [2]. In W7-AS both Alfvénic activity with frequencies of $100-250 \mathrm{kHz}$ (discharges \#43363) and low frequency activity with $f \sim 40 \div 70 \mathrm{kHz}$ (discharges \#30319 and \#34723) considerably reduced the global confinement time, see figures $4-6$. We select the W7AS discharge \#34723 and the NSTX discharge \#120438 for further consideration. Their characteristic parameters are given in table 1.

A single mode was responsible for thermal crashes in the W7-AS discharge \#34723. This mode was identified as Non-conventional Global Alfvén Eigenmode, NGAE, the mode in currentless plasmas with the frequency above a maximum of an Alfvén continuum branch (in contrast to the conventional Global Alfvén Eigenmode, GAE, having the frequency below a minimum of an Alfvén continuum branch) [4, 11, 14]. 


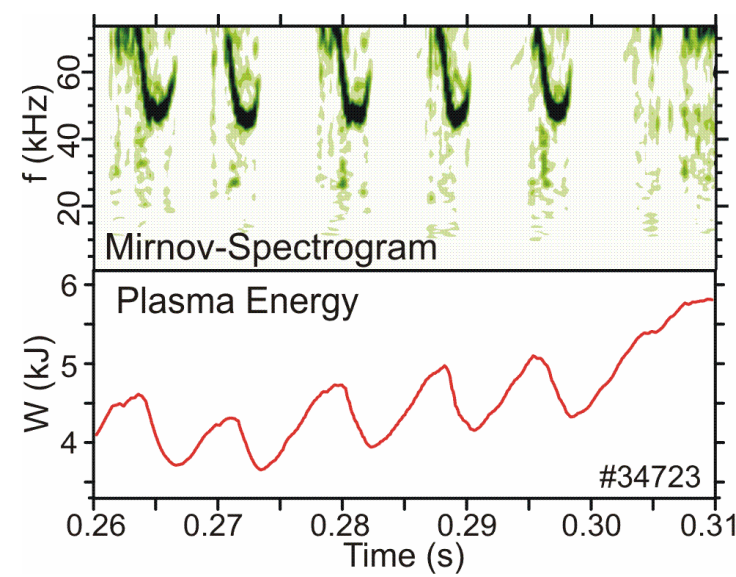

Figure 4. Bursting Alfvén instability in the Wendelstein 7-AS discharge \#34723. Thermal crashes occur at the end of the instability bursts, strong frequency chirping down takes place at the pre-crash state.
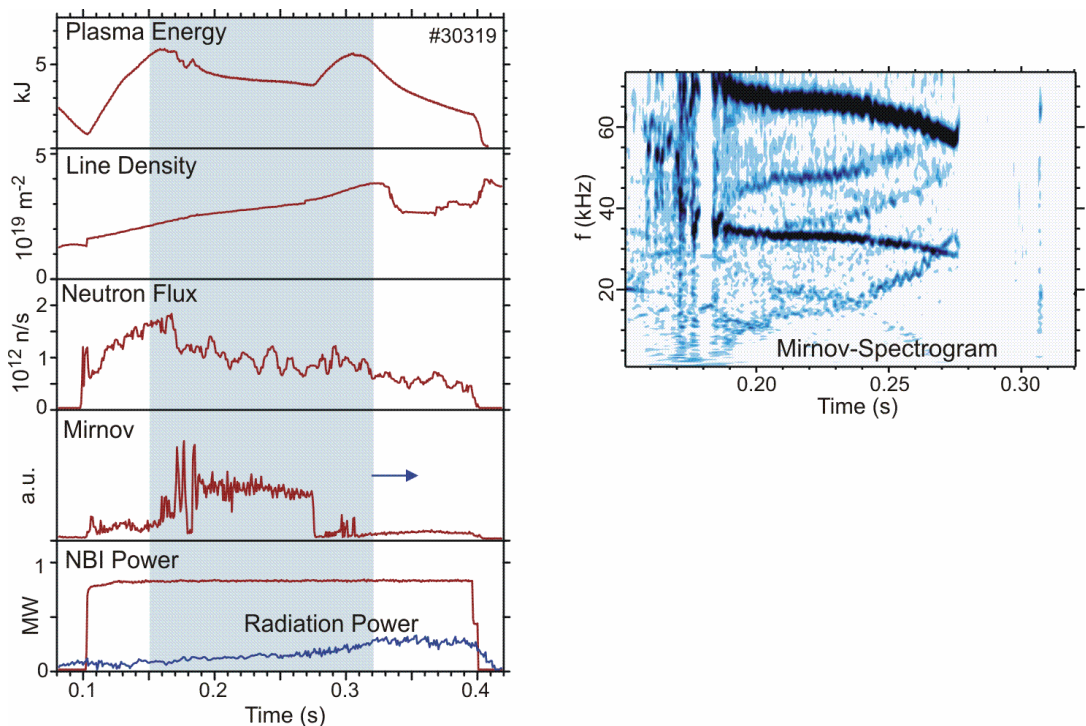

Figure 5. Deterioration of the energy confinement time in Wendelstein 7-AS during a quasi-steady-state low frequency Alfvén instability in the discharge \#30319.
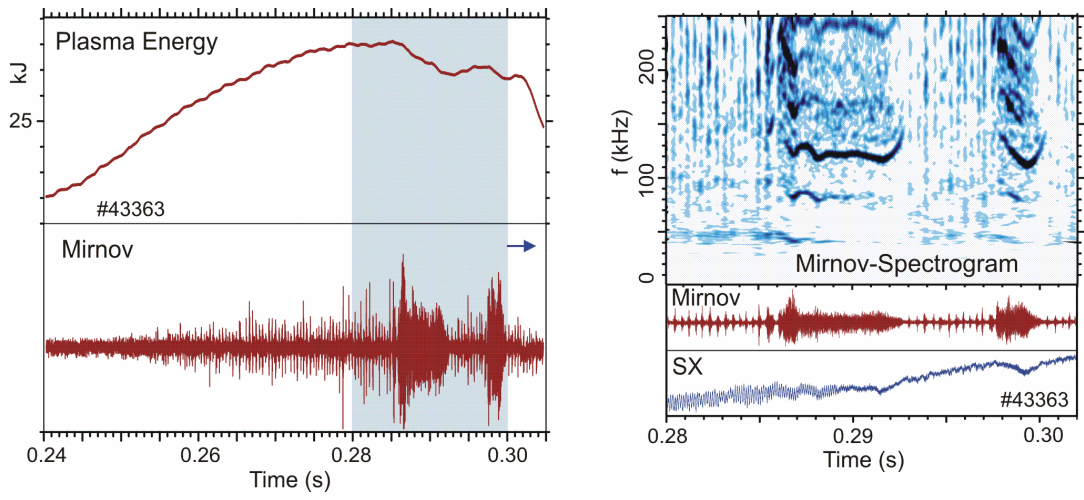

Figure 6. Quasi-steady-state multiple Alfvén modes accompanied by deterioration of the energy confinement time in Wendelstein 7-AS discharge \#43363. 
Table 1. Characteristic parameters at $r / a=1 / 3$ in the W7-AS discharge \#34723 and NSTX discharge \#120438. Notations: $A=R / a, \omega_{* \mathrm{e}}^{0}=m v_{\mathrm{e}}^{2} /\left(r \omega_{B} a\right), v_{\mathrm{e}}=\sqrt{T_{\mathrm{e}} / M_{\mathrm{e}}}$, $\nu_{\mathrm{e}}$ is the electron collision frequency, $t_{\mathrm{cr}}$ is the moment when the thermal crash started, $\omega_{\mathrm{b}}^{\mathrm{t}}=v_{\mathrm{e}} \sqrt{\epsilon_{\mathrm{t}}} /(\sqrt{2} q R), \epsilon_{\mathrm{t}}$ is the toroidal Fourier harmonic of the magnetic field, $\epsilon_{\mathrm{t}}=\epsilon$ in NSTX and $\epsilon_{\mathrm{t}} \approx \epsilon / 2$ in W7-AS [11], $z_{\mathrm{ef}}$ is the effective charge number.

\begin{tabular}{l|ccccccc}
\hline & $\mathrm{A}$ & $f, \mathrm{kHz}$ & $\mathrm{m} / \mathrm{n}$ & $\frac{\omega}{\omega_{\mathrm{b}}^{\mathrm{t}}}$ & $\frac{v_{\mathrm{A}}}{v_{\mathrm{e}}}$ & $\frac{\nu_{\mathrm{e}}}{k_{\|} v_{\mathrm{e}}}$ & $\frac{\omega}{\omega_{* e}^{0}}$ \\
\hline W7-AS & 13 & $45\left(t=t_{\mathrm{cr}}\right)$ & $5 / 2$ & 2.3 & 0.28 & 2.8 & 1.8 \\
NSTX & 1.2 & $\sim 800$ & $7 / 2$ & $\sim 1$ & 0.07 & $10^{-3} z_{\text {ef }}$ & 80 \\
\hline
\end{tabular}

The conditions of existence of NGAE modes in currentless stellarators differ from those for the Reversed Shear Alfvén Eigenmodes, RSAE, in tokamaks, although the RSAE frequency is located also above a maximum of the Alfvén continuum [14]. The observed NGAE instability had the frequency chirping down, the chirping occurred at the precrash stage of the instability bursts, see figure 4. On the other hand, the frequency chirping down, as shown in section 2, can be a consequence of the Doppler shift increasing in time. Thus, it is of interest to make corresponding estimates in order to see whether the observed drops of the frequency, $20 \div 25 \mathrm{kHz}$, can be explained by this mechanism. The NGAE mode was located in the region $r=3 \div 5 \mathrm{~cm}$, which implies that the energetic ions emitted plasmons in this region. The region of the wave damping was located at larger radii where a Kinetic Alfvén Wave (KAW) propagated. This implies that $r_{\mathrm{e}}>r_{\alpha}$ and, therefore, the Doppler shift can result in considerable chirping. To evaluate the effect, we note that for the Alfvén waves

$$
\frac{W}{n_{\mathrm{e}} T_{\mathrm{e}}}=\frac{2}{\beta_{\mathrm{e}}}\left(\frac{\widetilde{B}}{B}\right)^{2}
$$

where $\beta_{\mathrm{e}}=8 \pi n_{\mathrm{e}} T_{\mathrm{e}} / B^{2}$. We take $\beta_{\mathrm{e}}=0.7 \%\left(B=1.2 \mathrm{~T}, n_{\mathrm{e}}=10^{20} \mathrm{~m}^{-3}, T_{\mathrm{e}}=0.26 \mathrm{keV}\right)$ and $\widetilde{B} / B=3 \times 10^{-4}$ (this seems reasonable for a core localized NGAE because at the plasma edge $\widetilde{B}_{\vartheta}(a) / B=10^{-4} ; \widetilde{B}$ in the core region is not known from the experiment), which yields $W / n_{\mathrm{e}} T_{\mathrm{e}}=2.7 \times 10^{-5}$. Assuming that the duration of the pre-crash stage, $\tau^{\text {chirp}}$, is less than $\tau^{\text {vis }}$, we replace $\tau^{\text {vis }}$ with $\tau^{\text {chirp }}$ in equation (10) in order to evaluate the extra rotation velocity achieved for $\tau^{\text {chirp }} \approx 0.7 \mathrm{~ms}$. Then, taking into account that $\gamma_{\alpha} / \omega=2 \times 10^{-2}$ for the pre-crash stage [11] and $v_{\mathrm{i}}=1.15 \times 10^{7} \mathrm{~cm} \mathrm{~s}^{-1}$, we obtain $u_{\mathrm{i} \vartheta} / v_{\mathrm{i}} \sim 10^{-2}$ and $\Delta \omega=m u_{\mathrm{i} \vartheta} / r \sim-23 \mathrm{kHz}$, which agrees with the experiment. Probably, the Doppler shift caused by the instability-induced toroidal rotation is small because, first, $k_{\vartheta} \gg k_{\varphi}$ and, second, the mirror component of the equilibrium magnetic field, $\epsilon_{\mathrm{m}}$, was rather large in the core region.

Note that an alternative mechanism of the frequency chirping in the considered experiment is the evolution of equilibrium [11]. To conclude which one is most important or both are essential, a more detailed study is required.

Fourier harmonics of the equilibrium magnetic field in W7-AS are rather small, the largest ones (the mirror harmonic, $\epsilon_{\mathrm{m}}$, and the toroidal harmonic, $\epsilon_{\mathrm{t}}$ ) do not exceed 
$2.5 \times 10^{-2}$ in the region $r / a<0.5$ [11]. Therefore, the number of trapped particles is small. Furthermore, the locally trapped electrons weakly interact with the waves because $\omega \ll \omega_{\mathrm{b}}^{\text {loc }}$ ( $\omega_{\mathrm{b}}^{\text {loc }}$ is the bounce frequency of the locally trapped electrons). The circulating particles in W7-AS can strongly interact with the waves due to the resonance $\omega=k_{\|} v_{\|}$ because $v_{\mathrm{A}} / v_{\mathrm{e}}=v_{\|}^{\text {res }} / v_{\mathrm{e}}>\sqrt{\epsilon_{\mathrm{t}}}$. In contrast to this, $\omega=\omega_{\mathrm{b}}^{\mathrm{t}}, v_{\mathrm{A}} / v_{\mathrm{e}}=v_{\|}^{\text {res }} / v_{\mathrm{e}} \ll \sqrt{\epsilon}$, and the number of the trapped particles is large in NSTX. This implies that wave-particle resonance interaction involves mainly circulating electrons in W7-AS, whereas mainly trapped electrons interact with the waves and may play the main role in the observed anomalous transport in NSTX. Another important difference between the W7-AS and NSTX experiments is associated with the very large ratio of $\omega / \omega_{*}$ in NSTX. Because of this, the anomalous heat conductivity can hardly be responsible for anomalous electron transport in NSTX. However, in W7-AS the heat conductivity is sufficiently large, especially due to strong collisions $\left(\nu_{\mathrm{e}}>k_{\|} v_{\mathrm{e}}\right)$ and can result in thermal crashes [4].

Let us evaluate $\chi_{\mathrm{e}}$ in NSTX. Equations (27) and (29), which determine the heat conductivity, cannot be used because $\widetilde{E}_{\|}$is not known from the experiment. Instead, the line-averaged fluctuation amplitude of the electron density, $\left\langle\widetilde{n}_{\mathrm{e}}\right\rangle /\left\langle n_{\mathrm{e}}\right\rangle=1.5 \times 10^{-4}$, is known [3]. Therefore, we have to express $\widetilde{E}_{\|}$through $\widetilde{n}$. Note that $\widetilde{E}_{\|}=0$ in the ideal MHD approximation. However, in reality it never vanishes. As one can see from equation (25), $\widetilde{E}_{\|}$is considerable for the high frequency MHD modes having $k_{\|} q R \gg 1$, which was the case in the NSTX experiments where the mode frequencies reached 1.1 MHz. In addition, it is likely that KAWs generated at points of the local Alfvén resonances associated with GAE satellite harmonics considerably contribute to formation of $\widetilde{E}_{\|}$.

For the Alfvén waves $\widetilde{n}_{\mathrm{e}} \approx \widetilde{n}_{\mathrm{i}}$; the magnitude $\widetilde{n}_{\mathrm{i}}$ is connected with the radial ion displacement, $\xi_{r}$, by the incompressibility condition, $\widetilde{n}+n_{0}^{\prime} \xi_{r}=0 ; \xi_{r}$ is connected with the perturbed poloidal electric field by the equation $c \widetilde{E}_{\vartheta}+\mathrm{i} \omega B \xi_{r}=0$. Using these equations and equation (25) and assuming that $\widetilde{E}_{\perp} \sim \widetilde{E}_{\vartheta}$, we obtain:

$$
\widetilde{E}_{\|}=\frac{\mathrm{i} \omega k_{\|}}{c k_{\vartheta}} k_{\perp}^{2} \rho_{\mathrm{s}}^{2} B L_{n} \frac{\widetilde{n_{\mathrm{e}}}}{n_{0}} .
$$

Now we can write equations (27) and (29) as

$$
\begin{aligned}
\chi_{\mathrm{e}}^{\mathrm{c}} & =\sum_{m, n} \frac{1}{4} k_{\perp}^{4} \rho_{\mathrm{s}}^{4} L_{n}^{2} \omega\left(\frac{v_{r}}{v_{\mathrm{e}}}\right)^{5}\left(\frac{\widetilde{n}_{\mathrm{e}}}{n_{0}}\right)^{2}, \\
\chi_{\mathrm{e}}^{\mathrm{t}} & =\sum_{m, n} \Lambda k_{\perp}^{4} \rho_{\mathrm{s}}^{4} \frac{n^{2} q^{2}}{m^{2}} \frac{\omega^{2} L_{n}^{2}}{k_{\|} v_{\mathrm{e}}}\left(\frac{\widetilde{n}_{\mathrm{e}}}{n_{0}}\right)^{2} .
\end{aligned}
$$

Assuming that $\widetilde{n}_{\mathrm{e}}$ in the region of the instability exceeded $\left\langle\widetilde{n}_{\mathrm{e}}\right\rangle$ by a factor of three and taking into account that the modes with $n=2,3$ dominated in the experiment, we obtain that $\chi_{\mathrm{e}} \sim 1 \mathrm{~m}^{2} \mathrm{~s}^{-1}$, the main contribution coming from trapped particles (the estimates are made for $r / a=1 / 5$, where $\chi_{\mathrm{e}}$ calculated in reference [2] has a maximum). This is much less than the heat conductivity coefficient calculated by TRANSP, $\chi_{\mathrm{e}}^{\max }=200 \mathrm{~m}^{2} \mathrm{~s}^{-1}$ [2], which exceeds the Bohm diffusion coefficient, 
$D_{\mathrm{B}}=125 \mathrm{~m}^{2} \mathrm{~s}^{-1}$. This is not surprising: because $\omega \gg \omega_{* \mathrm{e}}$, the energy channelling and heat convection rather than the conductivity should play the main role. The code TRANSP ignoring these processes predicts $\chi_{\mathrm{e}}^{\text {ef }}$ defined by equation (4), which well overestimates the real heat conductivity coefficient.

Let us see whether the conditions of effective energy channelling are satisfied. First of all, a large fraction of the NBI (Neutral Beam Injection) power $\left[P_{\text {inj }}(r)\right]$ in the core region should be transferred to the waves. In NSTX $P_{\text {inj }}(0) \sim 1 \mathrm{MW} \mathrm{m}^{-3}$ when the total NBI power $\mathcal{P}_{\text {inj }}=6 \mathrm{MW}$. The beam power density transferred to the waves is

$$
P_{\alpha}=2 \gamma_{\alpha} W=2 \omega \frac{\gamma_{\alpha}}{\omega} \frac{B_{0}^{2}}{4 \pi} \frac{\widetilde{B}^{2}}{B_{0}^{2}},
$$

where $\widetilde{B} / B_{0}$ can be evaluated as $\widetilde{B} / B_{0} \sim k_{\|} L \widetilde{n}_{\mathrm{e}} / n_{\mathrm{e}} \sim 5 \times 10^{-3}$. Using this estimate and $B_{0}=0.45 \mathrm{~T}, f=1 \mathrm{MHz}$, we obtain that $P_{\alpha} \sim 1 \mathrm{MW} \mathrm{m}^{-3}$ for $\gamma_{\alpha} / \omega=2 \times 10^{-2}$, which is quite reasonable. It is clear that an energetic ion can be slowed down due to the interaction with the waves provided that the resonance region is suitable for this. The resonant velocities of the beam ions are determined by $\omega=\left[k_{\|} \pm s /(q R)\right] v_{\|}$, with $s$ integer, the $|s|>1$ resonances contributing considerably due to large orbit width of the beam ions [11]. Taking $|s|=0,1,2,3,4$ and $f=1 \mathrm{MHz}, v_{0} / v_{A}=2.9$ (with $v_{0}$ the birth velocity) we obtain the resonance velocities $v^{\text {res }} \chi / v_{0}=0.21,0.23,0.26,0.29,0.34,0.40,0.49,0.64,0.9$, where $\chi$ is the particle pitch angle. Thus, there are resonances of the $1 \mathrm{MHz}$ mode in the region $v^{\text {res }} / v_{0}=(0.22 \div 0.95) / \chi$. The presence of many modes with the frequencies from $0.5 \mathrm{MHz}$ to $1.1 \mathrm{MHz}$, which are observed experimentally, provides much more resonances. The resonance width can be evaluated from equation (30). This consideration enables us to conclude that the waves receive a large fraction of the injected energy.

The estimates above were made in the assumption that the radial position of the beam ions does not change during slowing down, which agrees with the experimental result showing no radial redistribution of the beam ions [2]. This agrees with our theory: Using equation (20) and assuming that a $90-\mathrm{keV}$ ion is slowed down to $\mathcal{E}=0$, we obtain that the ions are displaced negligibly; for instance, a particle born at $r / a=0.2$ will be displaced by $0.1 \mathrm{~cm}$.

Of course, specific calculations are required to see whether the energy channelling can indeed be responsible for radial profiles of the plasma temperature observed experimentally. Corresponding calculations based on a simple model were carried in reference [5]. They have demonstrated that the energy channelling seems to be a natural mechanism explaining the experimentally observed effects of instability on the temperature profile.

Note that an alternative explanation of the NSTX experiments was proposed in reference [15]. According to that work, these experiments can be explained by anomalous heat conductivity caused by stochastization of the magnetic field lines by multiple GAE instabilities. It is not clear, however, whether the plasma cooling due to the stochastization can exceed the plasma heating due to wave damping. Moreover, we 
think that this is unlikely: our theory (see section 3) predicts a restriction on the wave-induced heat flux, $\nabla \cdot \boldsymbol{q}_{\mathrm{e}}\left\langle Q_{\mathrm{e}}\right.$ when $\omega \gg \omega_{* \mathrm{e}}$ (except for a particular case of a monochromatic wave with $\Gamma_{\mathrm{e}}^{\mathrm{res}} \approx 0$ ). That is why we believe that the energy channelling is the only mechanism capable to explain the experiment. Nevertheless, heat convection and anomalous heat conductivity could also play an important role. They could not only contribute to the formation of the electron temperature profile but also be responsible for the rapid propagation of temperature perturbations produced by pellet injection an observation reported in references $[2,3]$.

There is a question of why instabilities in the frequency range of $0 \div 200 \mathrm{kHz}$ observed in the same NSTX discharge \#120438 had no influence on the electron temperature. To answer this question, it seems sufficient to consider wave-particle resonances. For the circulating particles the $s=0$ resonance with the Alfvénic perturbations does not

depend on the wave frequencies, $v_{\|}^{\text {res }} \approx v_{\mathrm{A}}$. Therefore, $v_{\|}^{\text {res }} / v_{\mathrm{e}} \approx 0.07 \ll 1$ and, thus, like in the case of the high frequency instabilities considered above, circulating electrons weakly interact with the waves. One can see that resonance interaction of trapped particles and low frequency perturbations involves also only electrons with very small energies. Indeed, $\omega=\omega_{\mathrm{b}}^{\mathrm{t}}$ for the $800 \mathrm{kHz}$ oscillations (see table 1). We infer from this that, e.g., the $100 \mathrm{kHz}$ perturbations interact with trapped electrons having the energy $\mathcal{E} / T_{\mathrm{e}}=1 / 64$.

\section{Summary and conclusions}

A theory of the electron transport and heating caused by energetic-ion-driven instabilities in toroidal plasmas is proposed, which is supplemented by a qualitative analysis. The obtained results can be summarized as follows.

It is shown that energetic-ion-driven instabilities can channel the energy and momentum of the energetic ions from the region where the energetic ions are located to the region where the destabilized modes are damped, see also [5]. This mechanism almost does not affect the radial redistribution of the energetic ions. It can be effective, in particular, when high frequency GAE modes are destabilized. In this case it leads to cooling the plasma core and heating the periphery. It is likely that the energy channelling was the main mechanism leading to the decrease of the electron temperature in the plasma centre with an increase of the injection power, which was observed in an experiment on NSTX [2]. Note that this energy channelling is a new phenomenon, it has nothing to do with the alpha channelling predicted earlier [16]: the alpha channelling does not redistribute the energy and momentum along the plasma radius; it leads to heating of ions rather than electrons.

According to the developed theory, there are two physical mechanisms responsible for the anomalous electron transport across the magnetic field. They are the anomalous heat conductivity and the heat convection caused by the waves. Based on bounceaveraged equations of the quasilinear theory, anomalous heat conductivity coefficients for the circulating particles and trapped particles are derived for the case of Alfvén 
instabilities. Conditions of their applicability are obtained.

It follows from our analysis that energetic-ion-driven instabilities can strongly affect the electron heat transport across the magnetic field, but their influence on the particle transport is small. This conclusion agrees with experimental observations on W7-AS and NSTX.

The factors responsible for the increase of the electron temperature at the periphery are revealed. They are the energy channelling, the acceleration of electrons during their motion outwards in the wave field, and the finite width of the instability region. In particular, due to the finite width of the instability region, the fluctuation amplitudes have a maximum at a certain radius, $r_{\mathrm{m}}$, vanishing at some radii for $r<r_{\mathrm{m}}$ and $r>r_{\mathrm{m}}$. Because of this, the instability leads to flattening of the originally peaked electron temperature, with the increase of $T_{\mathrm{e}}$ at $r>r_{\mathrm{m}}\left(\nabla \cdot \boldsymbol{q}_{\mathrm{e}}>0\right)$, where the fluctuation amplitudes decrease $\left(\nabla \cdot \boldsymbol{q}_{\mathrm{e}}<0\right)$.

It is concluded that energetic-ion-driven instabilities can lead to plasma rotation and concomitant frequency chirping. The rotation arises owing to momentum channelling. The increase of the rotation velocity after the beginning of the instability leads to a change of the Doppler shift, which implies that the development of the instability is accompanied by frequency chirping. The effect is larger when the waves are mainly emitted at a smaller radius than the radius of the wave absorption, in which case the frequency chirping down takes place.

It is emphasized that the generation of KAWs can play an important role, leading to anomalous electron heat transport beyond the region where the ideal MHD modes are located.

W7-AS and NSTX experiments where the electron transport was anomalous because of Alfvén instabilities excited by the energetic ions are considered. A discharge with strong thermal crashes in W7-AS and a discharge with the largest injected power and largest flattening of the electron profiles in NSTX were selected for analysis. It is concluded that different mechanisms are responsible for the anomalous phenomena in these facilities: it is the heat conductivity in W7-AS; they are the energy channelling and the heat convection in NSTX. It is found that circulating electrons are mainly affected by the waves in the W7-AS discharge, whereas trapped electrons are responsible for the effects observed in NSTX. The conclusion that mainly trapped electrons were influenced by the instabilities with $f \sim 1 \mathrm{MHz}$ in NSTX is actually supported by fact that no effects on the electrons were observed in the same discharge during the instabilities below $200 \mathrm{kHz}$, for which the wave-particle resonances are possible only for electrons of very low energies, $\mathcal{E} \ll T_{\mathrm{e}}$. It is found that strong frequency chirping down during the instability bursts in W7-AS can be explained by poloidal plasma rotation caused by the instability at the pre-crash stage.

It follows from our analysis that the key factor responsible for the influence of energetic-ion-driven instabilities on plasma heating, radial transport of electrons and plasma rotation is non-local momentum and energy exchange between the waves and particles of different species, which is a consequence of the fact that the regions of the 
wave emission and absorption are different. Depending on the magnitude of the local imbalance along the radius, the instabilities have either small or large influence on the bulk plasma properties.

\section{Acknowledgments}

This work is carried out within the STCU Project \#4588.

\section{Appendix A. The width of the resonance region for the particles trapped by Alfvén waves}

In this appendix we estimate the width of a resonance island in $v_{\|}$, which has been denoted above as $\delta v_{\|}^{\text {res }}$. To reach this aim, we derive an invariant of the particle motion in the electromagnetic field of a perturbation. For simplicity we neglect the plasma rotation and toroidicity. We assume that the perturbation consists of one harmonic $\propto \exp (\mathrm{i} \alpha)$ with $\alpha=m \vartheta-n \varphi-\omega t$ and is Alfvénic in the sense that the magnitude of the magnetic field is weakly perturbed, which gives us grounds to take $\tilde{\boldsymbol{A}}=\tilde{A}_{\|} \boldsymbol{b} \approx \tilde{A}_{\varphi} \nabla \phi$, where the tildes refer to the perturbation, $\boldsymbol{b}=\boldsymbol{B} / B$, and $\boldsymbol{A}$ is the vector potential. On the other hand, we take into account the non-zero longitudinal electric field of the wave (which is beyond the ideal MHD approximation). In what follows we assume that the co-ordinates $\vartheta$ and $\varphi$ are Boozer coordinates [18], which enables us to take the following simple gauge for the unperturbed vector potential [19]:

$$
A_{r 0}=0, \quad A_{\vartheta 0}=\psi, \quad A_{\varphi 0}=-\int \mathrm{d} \psi \iota(\psi),
$$

where $\psi=\psi(r)$ is the toroidal magnetic flux, $\iota$ is the rotational transform, the subscripts $r, \vartheta$ and $\varphi$ denote the corresponding co-variant components in the co-ordinate system $(r, \vartheta, \varphi)$, the subscript " 0 " refers to the equilibrium state.

If the aspect ratio of the plasma torus is large, the drift Lagrangian [17] in the co-ordinates $(r, \alpha, \phi)$ takes the form:

$$
\mathcal{L}=I_{\varphi} \dot{\varphi}+\frac{1}{m}\left(\frac{e}{c} A_{\vartheta}+M v_{\|} \frac{B_{\vartheta}}{B}\right) \dot{\alpha}+M v_{\|} \frac{B_{r}}{B} \dot{r}-H
$$

where the quantities $I_{\varphi}$ and $H$ given by

$$
\begin{aligned}
I_{\varphi} & =\frac{e}{c} A_{\varphi}+M v_{\|} R+\frac{n}{m}\left(\frac{e}{c} A_{\vartheta}+M v_{\|} \frac{B_{\vartheta}}{B}\right), \\
H & =\mathcal{E}+e \Phi-\frac{\omega}{m}\left(\frac{e}{c} A_{\vartheta}+M v_{\|} \frac{B_{\vartheta}}{B}\right)
\end{aligned}
$$

are constants of motion since the Lagrangian is independent on $\phi$ and $t, R$ is the major radius of the plasma torus, $\Phi$ is the scalar potential.

We use the invariants $I_{\varphi}$ and $H$ to study the resonant particle motion. We assume that $\Phi$ vanishes in the equilibrium state, $\Phi_{0}=0$ (our calculations with $\Phi_{0} \neq 0$ give the same estimate). One can show that the contribution of the terms containing 
$\tilde{\boldsymbol{B}}$ is negligible when the Larmor radius is sufficiently small. Then we simplify the invariants (A.3) and (A.4) as follows:

$$
\begin{aligned}
& \frac{e}{c}\left(\frac{n}{m}-\iota\right) \Delta \psi-\frac{e}{c} \iota^{\prime}(\Delta \psi)^{2}+M v_{\|} R+\frac{e}{c} \tilde{A}_{\varphi}=\text { const, } \\
& \frac{1}{2} M v_{\|}^{2}+e \tilde{\Phi}-\frac{\omega e}{m c} \Delta \psi=\mathrm{const},
\end{aligned}
$$

where $\Delta \psi=\psi-\psi_{\text {res }}$, the subscript "res" refers to the exact resonance $\omega=k_{\|} v_{\|}$, $k_{\|}=(m \iota-n) / R, \iota^{\prime}=\mathrm{d} \iota / \mathrm{d} \psi$, and we have restricted ourself to second-order terms while expanding equation (A.1) in $\Delta \psi$. Equations (A.5) and (A.6) are quadratic in $\Delta \psi$ and $v_{\|}$. They describe the structure of resonant islands in the phase space, the $\tilde{A}_{\varphi}$-term providing modulation depending on angles, the first-order terms relating the particle deviations in $\Delta \psi$ and $v_{\|}$, and the second-order terms determining the island width. For the thermal electrons, the main factor leading to detuning of a particle from the resonance is the particle acceleration (represented by the term $M v_{\|}^{2} / 2$ ) rather than the change of $k_{\|}$(represented by the $\iota^{\prime}$-term). Therefore, we neglect the latter term and exclude $\Delta \psi$ from these equations to obtain:

$$
\frac{1}{2} M\left(\Delta v_{\|}\right)^{2}+e \chi=\text { const, }
$$

where $\Delta v_{\|}=v_{\|}-v_{\| \text {res }}, v_{\| \text {res }}=\omega / k_{\|}$is the resonant longitudinal velocity, $\chi=$ $\tilde{\Phi}-\omega \tilde{A}_{\|} /\left(c k_{\|}\right)$so that $\tilde{E}_{\|}=-\nabla_{\|} \chi$.

Let us use equation (A.7) to find the resonance island width $\delta v_{\|}^{\text {res }}$. Obviously, $\chi_{\max }=-\chi_{\min }=\hat{E}_{\|} / k_{\|}$, where $\hat{E}_{\|}$is the amplitude of $\tilde{E}_{\|}, \chi_{\max }$ and $\chi_{\min }$ are the maximum and the minimum of $\chi$, respectively. As $\Delta v_{\|}=0$ at the X-point of the resonance island separatrix, we obtain the following equation for $\Delta v_{\| \max }$ (maximal $\Delta v_{\|}$ at the separatrix):

$$
\frac{1}{2} M\left(\Delta v_{\| \max }\right)^{2}+e \chi_{\min }=e \chi_{\max }
$$

whence we have

$$
\delta v_{\|}^{\mathrm{res}}=2 \Delta v_{\| \max }=4\left|\frac{e \hat{E}_{\|}}{M k_{\|}}\right|^{1 / 2} .
$$

Thus, in the assumptions we have made, the resonance width for the electromagnetic waves is exactly the same as for the potential waves.

\section{References}

[1] Weller A., Anton M., Geiger J. et al 2001 Phys. Plasmas 8931

[2] Stutman D., Tritz K., Delgado L. et al 2007 34th EPS Conf. on Plasma Physics (Warsaw, 2007) p2-061 http://epsppd.epfl.ch/Warsaw/pdf/P2_061.pdf.

[3] Stutman D., Delgado-Aparicio L., Gorelenkov N. et al 2009 Phys. Rev. Lett. 102115002

[4] Kolesnichenko Ya.I., Yakovenko Yu.V., Weller A. et al 2005 Phys. Rev. Lett. 94165004

[5] Kolesnichenko Ya.I., Yakovenko Yu.V. and Lutsenko V.V. 2010 Phys. Rev. Lett. 104075001

[6] Kolesnichenko Ya.I. 1980 Nucl. Fusion 20727 
[7] Belikov V.S. and Kolesnichenko Ya.I. 1982 Plasma Phys. 2461

[8] Kadomtsev B.B. 1964 Voprosy Teorii Plasmy (Reviews of Plasma Physics) vol 4 (Moscow: Atomizdat) 188 (in Russian)

[9] Belikov V.S. and Kolesnichenko Ya.I. 1987 Nucl. Fusion 271371

[10] Gorelenkov N.N., Belova E., Berk H.L. et al 2004 Phys. Plasmas 112586

[11] Kolesnichenko Ya.I., Lutsenko V.V., Weller A. et al 2006 Nucl. Fusion 46753

[12] Karney C.F.F. 1979 Phys. Fluids 222188

[13] Belikov V.S. and Kolesnichenko Ya.I. 1987 Nucl. Fusion 91371

[14] Kolesnichenko Ya.I., Lutsenko V.V., Weller A. et al 2007 Phys. Plasmas 14102504

[15] Gorelenkov N.N., Fredrickson E., Kaye S. et al 2009 Anomalous electron transport due to high frequency beam ion driven global Alfvén eigenmodes 11th IAEA Meeting on Energetic Particles in Magnetic Confinement Systems (Kyiv, Ukraine, 2009) invited talk IT-7

[16] Fisch N.J. and Herrmann M.C. 1999 Plasma Phys. Control. Fusion 41 A221

[17] Littlejohn R.G. 1983 J. Plasma Phys. 29111

[18] Boozer A.H. 1981 Phys. Fluids 241999

[19] White R. B. and Chance M.S. 1984 Phys. Fluids 272455 